\title{
Information-theoretic characterization of quantum chaos
}

\author{
Rüdiger Schackt and Carlton M. Caves \\ Center for Advanced Studies, Department of Physics and Astronomy, \\ University of New Mexico, Albuquerque, NM 87131-1156
}

(October 30, 2018)

\begin{abstract}
Hypersensitivity to perturbation is a criterion for chaos based on the question of how much information about a perturbing environment is needed to keep the entropy of a Hamiltonian system from increasing. We demonstrate numerically that hypersensitivity to perturbation is present in the following quantum maps: the quantum kicked top, the quantum baker's map, the quantum lazy baker's map, and the quantum sawtooth and cat maps.
\end{abstract}

\section{INTRODUCTION}

Present research on quantum chaos focuses mainly on what Berry [1] has called quantum chaology, namely "the study of semiclassical, but nonclassical, behavior characteristic of systems whose classical motion exhibits chaos." We pursue a different approach, which seeks to identify intrinsically chaotic features within quantum dynamics. A straightforward application of this approach, which attempts to generalize the classical notion of sensitivity to initial conditions, fails immediately, for two reasons: first, there is no quantum analogue of the classical phase-space trajectories in terms of which sensitivity to initial conditions is defined and, second, the unitarity of linear Schrödinger evolution precludes sensitivity to initial conditions in the quantum dynamics of state vectors. A classical Hamiltonian system has, however, in addition to its description in terms of trajectories governed by the Hamilton equations of motion, an equivalent description in terms of Liouville probability densities governed by the Liouville equation. Because it conserves phase-space volumes, the linear Liouville equation shares with the Schrödinger equation a lack of sensitivity to initial conditions. Again, in Berry's words [2], "the fact that this equation is linear and yet preserves the chaos of the trajectories disposes of the argument that the linearity of the Schrödinger equation is responsible for the absence of chaos in quantum mechanics." We have recently established a criterion for classical chaos in terms of Liouville probability densities [3 5], which we call hypersensitivity to perturbation and which has a straightforward quantum analogue [3, 6, 7].

Hypersensitivity to perturbation for a quantum system is defined in purely quantum terms, not in the semiclassical domain. Though quantum systems show no sensitivity to 
initial conditions, due to unitarity, they do show what one might call sensitivity to parameters in the Hamiltonian, as has been demonstrated for the quantum kicked top by Peres 8.9]. Our approach to quantum chaos could be viewed as a generalization of Peres's work: whereas Peres compares the time evolution of the same initial Hilbert-space vector for two slightly different parameter values in the kicked-top Hamiltonian, we study the distribution of Hilbert-space vectors arising from the many possible ways in which the Hamiltonian time evolution can be perturbed through interaction with an environment. Our approach, however, is embedded in a broader information-theoretic framework [3,5,7] that is quite different from the viewpoint expressed in [9].

In this paper we present numerical evidence that hypersensitivity to perturbation is present in a variety of quantum maps. We make no attempt to develop a general theory of quantum hypersensitivity to perturbation; rather, our attitude in this paper is exploratory: by investigating numerically the perturbed dynamics of several quantum systems, we seek to determine whether hypersensitivity to perturbation provides a criterion for distinguishing regular from chaotic dynamics in quantum systems. The paper is organized as follows. Section $\mathbb{1}$ defines hypersensitivity to perturbation and reviews the main results for classical Hamiltonian systems. Section [II introduces the model of the quantum kicked top interacting with a perturbing environment. Section $\mathbb{I V A}$ explains how hypersensitivity to perturbation can be related to the distribution of vectors in Hilbert space; Sec. IVB contains numerical results for the quantum kicked top. In Sec. $\mathrm{V}$ we present numerical evidence for hypersensitivity to perturbation in a variety of quantum maps on the unit square. Section $\nabla$ concludes with brief discussion.

\section{HYPERSENSITIVITY TO PERTURBATION}

Hypersensitivity to perturbation, in either classical or quantum mechanics, is defined in terms of information and entropy. The entropy $H$ of an isolated physical system (Gibbs entropy for a classical system, von Neumann entropy for a quantum system) does not change under Hamiltonian time evolution. If the time evolution of the system is perturbed through interaction with an incompletely known environment $\mathcal{E}$, however, averaging over the perturbation typically leads to an entropy increase $\Delta H_{\mathcal{S}}$. The increase of the system entropy can be limited to an amount $\Delta H_{\mathrm{tol}}$, the tolerable entropy increase, by obtaining, from the environment, information about the perturbation. We denote by $\Delta I_{\min }$ the minimum information about the environment needed, on the average, to keep the system entropy below the tolerable level $\Delta H_{\mathrm{tol}}$. A formal definition of the quantities $\Delta H_{\mathcal{S}}, \Delta H_{\mathrm{tol}}$, and $\Delta I_{\min }$ is given below.

Entropy and information acquire physical content in the presence of a heat reservoir at temperature $T$. If all energy in the form of heat is ultimately exchanged with the heat reservoir, then each bit of entropy, i.e., each bit of missing information about the system state, reduces by the amount $k_{B} T \ln 2$ the energy that can be extracted from the system in the form of useful work. The connection between acquired information and work is provided by Landauer's principle [10,11, according to which not only each bit of missing information, but also each bit of acquired information, has a free-energy cost of $k_{B} T \ln 2$. This cost, the Landauer erasure cost, is payed when the acquired information is erased. Acquired information can be quantified by algorithmic information [12 [16]. 
We are now in a position to define hypersensitivity to perturbation. We say that a system is hypersensitive to perturbation if the information $\Delta I_{\min }$ required to reduce the system entropy from $\Delta H_{S}$ to $\Delta H_{\text {tol }}$ is large compared to the entropy reduction $\Delta H_{S}-\Delta H_{\text {tol }}$, i.e.,

$$
\frac{\Delta I_{\text {min }}}{\Delta H_{S}-\Delta H_{\text {tol }}} \gg 1 \text {. }
$$

The information $\Delta I_{\min }$ purchases a reduction $\Delta H_{S}-\Delta H_{\text {tol }}$ in system entropy, which is equivalent to an increase in the useful work that can be extracted from the system; hypersensitivity to perturbation means that the Landauer erasure cost of the information is much larger than the increase in available work.

Hypersensitivity to perturbation means that the inequality (2.1) holds for almost all values of $\Delta H_{\mathrm{tol}}$. The inequality (2.1) tends always to hold, however, for sufficiently small values of $\Delta H_{\mathrm{tol}}$. The reason is that for these small values of $\Delta H_{\mathrm{tol}}$, one is gathering enough information from the perturbing environment to track a particular system state whose entropy is nearly equal to the initial system entropy. In other words, one is essentially tracking a particular realization of the perturbation among all possible realizations. Thus, for small values of $\Delta H_{\mathrm{tol}}$, the information $\Delta I_{\min }$ becomes a property of the perturbation; it is the information needed to specify a particular realization of the perturbation. The important regime for assessing hypersensitivity to perturbation is where $\Delta H_{\text {tol }}$ is fairly close to $\Delta H_{S}$, and it is in this regime that one can hope that $\Delta I_{\min }$ reveals something about the system dynamics, rather than properties of the perturbation.

In [5] we have shown that a large class of classically chaotic systems shows exponential hypersensitivity to perturbation, which is characterized by an exponential growth with time of the ratio (2.1) between information and entropy reduction, for a broad range of values of $\Delta H_{\text {tol }}$ below $\Delta H_{S}$. The exponential rate of growth is determined by the Kolmogorov-Sinai entropy of the classical dynamics. In preliminary work [6, [] we have applied the notion of hypersensitivity to perturbation to quantum systems and, through numerical simulations, have shown that hypersensitivity to perturbation seems to distinguish regular from chaotic quantum dynamics. The present paper contains a more detailed treatment of hypersensitivity to perturbation, including numerical evidence for a range of prototypic quantum systems.

To define the quantities $\Delta H_{\mathcal{S}}, \Delta H_{\text {tol }}$, and $\Delta I_{\text {min }}$ rigorously in the quantum case, consider a quantum system $\mathcal{S}$ coupled to an environment $\mathcal{E}$. We assume that the total system $\mathcal{S} \otimes \mathcal{E}$ is initially in a product state

$$
\hat{\rho}_{\text {total }}(t=0)=\left|\psi_{0}\right\rangle\left\langle\psi_{0}\right| \otimes \hat{\rho}_{\mathcal{E}},
$$

combining a pure system state $\left|\psi_{0}\right\rangle$ with a mixed environment state $\hat{\rho}_{\mathcal{E}}$. The initial von Neumann entropy $H$ of the system is zero. After evolving for a time $t$ under the action of the total Hamiltonian $\hat{H}_{\text {total }}$, the total system is described by a density operator $\hat{\rho}_{\text {total }}(t)$, which is generally not a product state. The state $\hat{\rho}_{\mathcal{S}}$ of the system $\mathcal{S}$ alone at time $t$ is then obtained by tracing over the environment,

$$
\hat{\rho}_{\mathcal{S}}=\operatorname{tr}_{\mathcal{E}}\left(\hat{\rho}_{\text {total }}(t)\right) .
$$

The increase in system entropy due to tracing over the environment is given by 


$$
\Delta H_{\mathcal{S}}=-\operatorname{tr}_{\mathcal{S}}\left(\hat{\rho}_{\mathcal{S}} \log _{2} \hat{\rho}_{\mathcal{S}}\right) .
$$

Now suppose a measurement is performed on the environment $\mathcal{E}$. The most general measurement is described by a positive-operator-valued measure (POVM) [9,17]. We assume for convenience that the measurement is described by a POVM that has a discrete set of possible outcomes $r$ with probabilities $p_{r}$. For each measurement outcome $r$, we denote by $\hat{\rho}_{r}$ the system state conditional on the measurement outcome $r$; the conditional system states obey the relation (for a justification, see Appendix A)

$$
\sum_{r} p_{r} \hat{\rho}_{r}=\hat{\rho}_{\mathcal{S}} .
$$

We further define the system entropy conditional on measurement outcome $r$,

$$
\Delta H_{r}=-\operatorname{tr}_{\mathcal{S}}\left(\hat{\rho}_{r} \log _{2} \hat{\rho}_{r}\right),
$$

the average conditional entropy

$$
\Delta H=\sum_{r} p_{r} \Delta H_{r},
$$

and the average information

$$
\Delta I=-\sum_{r} p_{r} \log _{2} p_{r} .
$$

Actually $\Delta I$ is a lower bound to the average algorithmic information needed to specify the measurement outcome $r$. One of us has shown [16], however, that the minimum average algorithmic information can be bounded above by $\Delta I+1$, so that $\Delta I$ is an excellent approximation to the minimum average algorithmic information.

The minimum amount of information about the perturbing environment to keep the system entropy from increasing by more than the tolerable amount $\Delta H_{\text {tol }}$ can now be defined as

$$
\Delta I_{\min }=\inf _{\Delta H \leq \Delta H_{\mathrm{tol}}} \Delta I,
$$

where the infimum is taken over all environment measurements for which the average conditional entropy increase $\Delta H$ does not exceed $\Delta H_{\text {tol }}$. In other words, $\Delta I_{\min }$ is the information about the environment it takes to reduce the entropy increase of the system from $\Delta H_{\mathcal{S}}$ (the increase due to averaging over the perturbation) down to $\Delta H_{\text {tol }}$; i.e., $\Delta I_{\min }$ is the minimum information about the environment needed to purchase a reduction $\Delta H_{\mathcal{S}}-\Delta H_{\text {tol }}$ in system entropy.

\section{QUANTUM KICKED TOP}

\section{A. Kicked-top Hamiltonian}

The quantum model of the kicked top [18,[19] describes a spin- $J$ particle - i.e., an angular momentum vector $\hbar \hat{\mathbf{J}}=\hbar\left(\hat{J}_{x}, \hat{J}_{y}, \hat{J}_{z}\right)$, where $\left[\hat{J}_{i}, \hat{J}_{j}\right]=i \epsilon_{i j k} \hat{J}_{k}$-whose dynamics in $(2 J+1)$ dimensional Hilbert space is governed by the Hamiltonian 


$$
\hat{H}_{\text {top }}(t)=(\hbar p / T) \hat{J}_{z}+(\hbar k / 2 J) \hat{J}_{x}^{2} \sum_{n=-\infty}^{+\infty} \delta(t-n T) .
$$

The free precession of the spin around the $z$ axis (first term in the Hamiltonian) is interrupted periodically by sudden kicks or twists at times $n T$ with twist parameter $k$ (second term in the Hamiltonian). The angle of free precession between kicks is given by $p$. In this paper we use $p=\pi / 2$.

We look at the time evolution of an initial Hilbert-space vector $\left|\psi_{0}\right\rangle$ at discrete times $n T$. After $n$ time steps, the evolved vector is given by

$$
\left|\psi_{n}\right\rangle=\hat{T}_{\text {top }}^{n}\left|\psi_{0}\right\rangle
$$

where $\hat{T}_{\text {top }}$ is the unitary Floquet operator

$$
\hat{T}_{\text {top }}=e^{-i(k / 2 J) \hat{J}_{x}^{2}} e^{-i \pi \hat{J}_{z} / 2} .
$$

The model has a conserved parity, which for half-integer $J$ takes the form

$$
\hat{S}=-i e^{-i \pi \hat{J}_{z}}
$$

and which permits factorization of the matrix representation of the operator $\hat{T}_{\text {top }}$ into two blocks. Starting from a state with definite parity, the whole dynamical evolution occurs in the invariant Hilbert subspace with the given parity. For half-integer $J$, the dimension of the even-parity subspace (eigenspace of $\hat{S}$ with eigenvalue 1 ) is $J+\frac{1}{2}$. In this paper, we work with $J=511.5$ in the 512-dimensional even-parity subspace or with $J=63.5$ in the 64-dimensional even-parity subspace. We consider only the projection of the initial vector in the even subspace. Numerical evidence and symmetry considerations [8] suggest that no additional insight is gained by including the odd-parity subspace. In any case, the restricted model can be regarded as a quantum map in its own right, which can be investigated independently of the behavior of the complete kicked-top model.

\section{B. Choice of initial vector}

Depending on the initial condition, the classical map corresponding to the Floquet operator (3.3) displays regular as well as chaotic behavior [19]. Following [8], we choose initial Hilbert-space vectors for the quantum evolution that correspond to classical initial conditions located in regular and chaotic regions of the classical dynamics, respectively.

The classical map corresponding to the quantum map (3.3) is obtained by introducing the unit vector $(X, Y, Z) \equiv \hat{\mathbf{J}} / J$ and performing the limit $J \rightarrow \infty$. One obtains [19]

$$
\begin{aligned}
& X^{\prime}=-Y, \\
& Y^{\prime}=X \cos k Y+Z \sin k Y, \\
& Z^{\prime}=Z \cos k Y-X \sin k Y,
\end{aligned}
$$

which is an area-preserving map of the unit sphere, i.e., an area-preserving map on the configuration space of a classical spin with fixed magnitude. Depending on the value of the 
twist parameter $k$, this map has regions of chaotic behavior interspersed with regular regions associated with elliptic cyclic points. In this paper, we are interested in two cyclic points of the map for $k=3$. One is an elliptic fixed point of period 1, located at [20]

$$
Z=\cos \theta=0.455719, \varphi=3 \pi / 4
$$

where we use spherical coördinates $\theta=\cos ^{-1} Z$ and $\varphi=\tan ^{-1}(Y / X)$. For $k=3$ the elliptic fixed point (3.6) is surrounded by an oval-shaped regular region of regular dynamics, extending about 0.3 radians in the $\varphi$-direction and about 0.5 radians in the $\theta$-direction. The other cyclic point of interest to us is a hyperbolic fixed point of period 4, which has a positive Lyapunov exponent. It is located at 20]

$$
Z=\cos \theta=0.615950, \varphi=\pi / 4
$$

in the middle of a chaotic region

We want to choose initial vectors for the quantum evolution that correspond as closely as possible to the classical directions (3.6) and (3.7). For this purpose, we use coherent states $|\theta, \varphi\rangle$ [21] 23], defined by the relation

$$
\mathbf{n} \cdot \hat{\mathbf{J}}|\theta, \varphi\rangle=J|\theta, \varphi\rangle
$$

where $\mathbf{n}$ is the unit vector pointing in the direction given by $\theta$ and $\varphi$. All coherent states can be generated by an appropriate rotation of the state $|J, M=J\rangle=|\theta=0, \varphi=0\rangle$, where $|J, M\rangle(M=-J, \ldots, J)$ is the simultaneous eigenstate of $\hat{J}^{2}$ and $\hat{J}_{z}$ with eigenvalues $J(J+1)$ and $M$, respectively.

The distance between two normalized vectors $\left|\psi_{1}\right\rangle$ and $\left|\psi_{2}\right\rangle$ is defined as the Hilbert-space angle

$$
s\left(\left|\psi_{1}\right\rangle,\left|\psi_{2}\right\rangle\right)=\cos ^{-1}\left(\left|\left\langle\psi_{1} \mid \psi_{2}\right\rangle\right|\right)
$$

between the two vectors [24]. Consider two coherent states $|\theta, \varphi\rangle$ and $\left|\theta^{\prime}, \varphi^{\prime}\right\rangle$. In terms of the angle $\alpha$ between the directions $(\theta, \varphi)$ and $\left(\theta^{\prime}, \varphi^{\prime}\right)$, the distance between the two coherent states is given by 25

$$
\cos \left[s\left(|\theta, \varphi\rangle,\left|\theta^{\prime}, \varphi^{\prime}\right\rangle\right)\right]=\left|\left\langle\theta, \varphi \mid \theta^{\prime}, \varphi^{\prime}\right\rangle\right|=[\cos (\alpha / 2)]^{2 J} \simeq \exp \left(-J \alpha^{2} / 4\right),
$$

where the approximation is valid for large $J$. Two coherent states can therefore be regarded as roughly orthogonal if $\alpha>2 J^{-1 / 2}[8]$. The size of the coherent state $|\theta, \varphi\rangle$ is conveniently defined in terms of the $Q$ function

$$
Q_{\theta, \varphi}\left(\theta^{\prime}, \varphi^{\prime}\right) \equiv\left|\left\langle\theta^{\prime}, \varphi^{\prime} \mid \theta, \varphi\right\rangle\right|^{2}=[\cos (\alpha / 2)]^{4 J} \equiv Q(\alpha)
$$

Since $Q\left(2 J^{-1 / 2}\right) \simeq e^{-2} Q(0)$, the $Q$ function of the coherent state $|\theta, \varphi\rangle$ is very small outside a region of radius $2 J^{-1 / 2}$ centered at the direction $(\theta, \varphi)$. For the value $J=511.5$ used in this paper to study regular behavior, one finds a radius of $2 J^{-1 / 2} \simeq 0.09$ radians, less than the size of the regular region around the elliptic fixed point (3.6). 


\section{Interaction with an environment}

Suppose now that the kicked top interacts with an environment consisting of a collection of degenerate two-state systems. For the interaction Hamiltonian we assume the form

$$
\hat{H}_{\mathrm{int}}=\hbar g \hat{J}_{z} \sum_{n=-\infty}^{+\infty}\left(\hat{\sigma}_{3}\right)_{n} \delta(t-n T-\epsilon) .
$$

Here $\left(\hat{\sigma}_{3}\right)_{n}$ is a Pauli operator for the $n$th two-state system, $g$ is the interaction strength, and $\epsilon$ is an infinitesimal time interval. The kicked top thus interacts with a single environment system at a time, interacting with the $n$th two-state system just after the $n$th twist, i.e., just after the $n$th unperturbed time step. The $n$th perturbed time step includes the $n$th unperturbed time step and the immediately subsequent coupling to the $n$th two-state system. The total Hamiltonian for system and environment is

$$
\hat{H}_{\text {total }}=\hat{H}_{\text {top }}+\hat{H}_{\text {int }}
$$

where $\hat{H}_{\text {top }}$ is given by Eq. (3.1) with $p=\pi / 2$.

As was explained in Sec. [I], we take the initial state of the total system to be of the form $\left|\psi_{0}\right\rangle\left\langle\psi_{0}\right| \otimes \hat{\rho}_{\mathcal{E}}$, where here the initial state $\left|\psi_{0}\right\rangle$ of the system is a coherent state centered at one of the fixed points (3.6) and (3.7) and the initial state of the environment, for the $n$ two-state systems that are relevant for the first $n$ time steps, is

$$
\hat{\rho}_{\mathcal{E}}=\sum_{l_{1}, \ldots, l_{n} \in\{-1,+1\}} 2^{-n}\left|l_{1}\right\rangle \cdots\left|l_{n}\right\rangle\left\langle l_{n}\right| \cdots\left\langle l_{1}\right|
$$

Each of the environment oscillators is with equal probability in the "lower" state $|-1\rangle$, where $\hat{\sigma}_{3}|-1\rangle=-|-1\rangle$, or in the "upper" state $|+1\rangle$, where $\hat{\sigma}_{3}|+1\rangle=|+1\rangle$.

Now suppose the kicked top and the environment evolve together for $n$ time steps. We want to make a measurement on the environment in order to reduce the entropy increase of the system. The form of the interaction Hamiltonian (3.12) suggests that the natural basis for a measurement of the environment state is the simultaneous eigenbasis of the operators $\left(\hat{\sigma}_{3}\right)_{m}$. If the environment is measured in this basis and is found to be in the state $\left|l_{1}\right\rangle \cdots\left|l_{n}\right\rangle$ $\left(l_{m} \in\{-1,+1\}, m=1, \ldots, n\right)$, then the system state after $n$ perturbed steps is given by

$$
\left|\psi_{n}\right\rangle=\hat{T}_{\text {top }}\left(l_{n}\right) \cdots \hat{T}_{\text {top }}\left(l_{1}\right)\left|\psi_{0}\right\rangle
$$

where

$$
\hat{T}_{\text {top }}\left(l_{m}\right)=e^{-i g l_{m} \hat{J}_{z}} e^{-i(k / 2 J) \hat{J}_{x}^{2}} e^{-i \pi \hat{J}_{z} / 2}=e^{-i g l_{m} \hat{J}_{z}} \hat{T}_{\text {top }}
$$

is the unperturbed Floquet operator (3.3) followed by an additional rotation about the $z$ axis by angle $g l_{m}$. Notice that $\hat{T}_{\text {top }}\left(l_{m}\right)$ commutes with parity (3.4) and, hence, does not couple odd- and even-parity subspaces.

The effect of the coupling to the environment, together with the measurement, is to produce a binary stochastic perturbation at the end of each time step. The top rotates by $+g$ if the two-state system is found in the upper state and by $-g$ if it is found in the lower state, the two possibilities occurring with equal probability. After $n$ steps, there are $2^{n}$ 
different equally probable measurement results, each leading to a pure system state. Since there is no simple commutation relation between the operators $\hat{T}_{\text {top }}(-1)$ and $\hat{T}_{\text {top }}(+1)$, these $2^{n}$ system states are all different. Any measurement that fails to distinguish completely between different environment states $\left|l_{1}\right\rangle \cdots\left|l_{n}\right\rangle$ leads therefore to a mixed system state. To keep the system state pure, one has to specify which of the $2^{n}$ equally probable alternatives is realized. The minimal information about the environment needed to keep the system entropy at the level $\Delta H_{\text {tol }}=0$ is therefore $\Delta I_{\min }=n$ bits. This means that the information about the environment needed to keep track of the exact system state is unbounded as the number of steps increases, an effect that is independent of the exact nature of the system dynamics.

The value of $\Delta I_{\min }$ for $\Delta H_{\mathrm{tol}}=0$, therefore, is not a useful measure of the difficulty in keeping the system entropy small. We must determine how fast $\Delta I_{\text {min }}$ decreases if $\Delta H_{\text {tol }}$ is increased. Unfortunately, it is difficult to find an optimal measurement leading to minimal information for an arbitrary value of $\Delta H_{\text {tol }}$. It is possible, however, to find a nearly optimal measurement based on the following idea. The interaction with the environment (3.12) is of a special form that can be described in terms of a randomly perturbed unitary evolution operator. Each of the $2^{n}$ possible measurement results corresponds to one of $2^{n}$ different perturbation histories obtained by applying every possible sequence of perturbed unitary evolution operators $\hat{T}_{\text {top }}(-1)$ and $\hat{T}_{\text {top }}(+1)$ for $n$ steps. Consider the list $\mathcal{L}$ of $2^{n}$ vectors generated by all perturbation histories. In the next section, we show how a nearly optimal measurement can be found in the class of measurements whose outcomes partition the list $\mathcal{L}$ into groups of vectors.

\section{DISTRIBUTION OF VECTORS IN HILBERT SPACE}

From this section on, we always assume that the interaction with the environment can be described by a stochastic unitary time evolution. In the first part of this section, we explore the relation between hypersensitivity to perturbation and the distribution of the vectors in Hilbert space resulting from different realizations of the stochastic evolution. In the second part, we use this relation to investigate hypersensitivity to perturbation in the quantum kicked top.

\section{A. General theory}

Given the assumption of a stochastic unitary time evolution, the interaction with the environment at a given time can be described by a list $\mathcal{L}=\left(\left|\psi_{1}\right\rangle, \ldots,\left|\psi_{N}\right\rangle\right)$ of $N$ vectors in $D$-dimensional Hilbert space, together with probabilities $q_{1}, \ldots, q_{N}$, each vector in the list corresponding to a particular realization of the perturbation, which we call a perturbation history. Averaging over the perturbing environment leads to a system density operator

$$
\hat{\rho}_{\mathcal{S}}=\sum_{j=1}^{N} q_{j}\left|\psi_{j}\right\rangle\left\langle\psi_{j}\right|,
$$

with entropy $\Delta H_{\mathcal{S}}$ as given by Eq. (2.4). Consider the class of measurements on the environment whose outcomes partition the list $\mathcal{L}$ into $R$ groups, labeled by $r=1, \ldots, R$. We 
denote by $N_{r}$ the number of vectors in the $r$ th group $\left(\sum_{r=1}^{R} N_{r}=N\right)$. The $N_{r}$ vectors in the $r$ th group and their probabilities are denoted by $\left|\psi_{1}^{r}\right\rangle, \ldots,\left|\psi_{N_{r}}^{r}\right\rangle$ and $q_{1}^{r}, \ldots, q_{N_{r}}^{r}$, respectively. The measurement outcome $r$, occurring with probability

$$
p_{r}=\sum_{i=1}^{N_{r}} q_{i}^{r},
$$

indicates that the system state is in the $r$ th group. The system state conditional on the measurement outcome $r$ is described by the density operator

$$
\hat{\rho}_{r}=p_{r}^{-1} \sum_{i=1}^{N_{r}} q_{i}^{r}\left|\psi_{i}^{r}\right\rangle\left\langle\psi_{i}^{r}\right| .
$$

Using Eqs. (4.2) and (4.3), we define the conditional system entropy $\Delta H_{r}$, the average conditional entropy $\Delta H$, and the average information $\Delta I$ as in Eqs. (2.6)-(2.8).

We now describe nearly optimal measurements, i.e., nearly optimal groupings, in the sense of the discussion in Sec. III. Given a tolerable entropy $\Delta H_{\text {tol }}$, we want to partition the list of vectors $\mathcal{L}$ into groups so as to minimize the information $\Delta I$ without violating the condition $\Delta H \leq \Delta H_{\text {tol }}$. To minimize $\Delta I$, it is favorable to make the groups as large as possible. Furthermore, to reduce the contribution to $\Delta H$ of a group containing a given number of vectors, it is favorable to choose vectors that are as close together as possible in Hilbert space. Here the distance between two vectors $\left|\psi_{1}\right\rangle$ and $\left|\psi_{2}\right\rangle$ can be quantified in terms of the Hilbert-space angle (3.9). Consequently, to find a nearly optimal grouping, we choose an arbitrary resolution angle $\phi(0 \leq \phi \leq \pi / 2)$ and group together vectors that are less than an angle $\phi$ apart. More precisely, groups are formed in the following way. Starting with the first vector $\left|\psi_{1}\right\rangle$ in the list $\mathcal{L}$, the first group is formed of $\left|\psi_{1}\right\rangle$ and all vectors in $\mathcal{L}$ that are within an angle $\phi$ of $\left|\psi_{1}\right\rangle$. The same procedure is repeated with the remaining vectors to form the second group, then the third group, continuing until no ungrouped vectors are left. This grouping of vectors corresponds to a partial averaging over the perturbations. To describe a vector at resolution level $\phi$ amounts to averaging over those details of the perturbation that do not change the final vector by more than an angle $\phi$.

For each resolution angle $\phi$, the grouping procedure described above defines an average conditional entropy $\Delta H \equiv \Delta H(\phi)$ and an average information $\Delta I \equiv \Delta I(\phi)$. If we choose, for a given $\phi$, the tolerable entropy $\Delta H_{\text {tol }}=\Delta H(\phi)$, then to a good approximation, the information $\Delta I_{\min }$ is given by $\Delta I_{\min } \simeq \Delta I(\phi)$. By determining the entropy $\Delta H(\phi)$ and the information $\Delta I(\phi)$ as functions of the resolution angle $\phi$, there emerges a rather detailed picture of how the vectors are distributed in Hilbert space. If $\Delta I(\phi)$ is plotted as a function of $\Delta H(\phi)$ by eliminating the angle $\phi$, one obtains a good approximation to the functional relationship between $\Delta I_{\min }$ and $\Delta H_{\text {tol }}$.

It is easy to see that information and entropy obey the inequalities

$$
\begin{gathered}
\Delta I(0) \geq \Delta I(\phi) \geq \Delta I(\pi / 2)=0, \\
0=\Delta H(0) \leq \Delta H(\phi) \leq \Delta H(\pi / 2) .
\end{gathered}
$$

The first of the information inequalities in Eq. (4.4) follows from the fact that any group at resolution $\phi$ is the union of groups at resolution $\phi=0$; in words, the average information 
needed to specify a group at resolution $\phi=0$ is equal to the average information needed to specify a group at resolution $\phi$ plus the average information needed to specify $\phi=0$ groups within the groups at resolution $\phi$. The latter of the entropy inequalities in Eq. (4.5) is a consequence of the concavity of the von Neumann entropy 26,27. A general theorem about average density operators [26,27] shows that, for all $\phi$,

$$
\Delta I(\phi)+\Delta H(\phi) \geq \Delta H(\pi / 2) .
$$

In general, $\Delta I(\phi)$ is a decreasing function of $\phi$, whereas $\Delta H(\phi)$ is increasing. This monotonicity can sometimes be violated, however, because of discontinuous changes in the grouping of vectors.

As a further characterization of our list of vectors, we calculate the distribution $g(\phi)$ of Hilbert-space angles $\phi=s\left(|\psi\rangle,\left|\psi^{\prime}\right\rangle\right)=\cos ^{-1}\left(\left|\left\langle\psi \mid \psi^{\prime}\right\rangle\right|\right)$ between all pairs of vectors $|\psi\rangle$ and $\left|\psi^{\prime}\right\rangle$. For vectors distributed randomly in $D$-dimensional Hilbert space, the distribution function $g(\phi)$ is given by [7]

$$
g(\phi)=\frac{d \mathcal{V}_{D}(\phi) / d \phi}{\mathcal{V}_{D}}=2(D-1)(\sin \phi)^{2 D-3} \cos \phi
$$

Here $\mathcal{V}_{D}(\phi)=(\sin \phi)^{2(D-1)} \mathcal{V}_{D}$ is the volume contained within a sphere of radius $\phi$ in $D$ dimensional Hilbert space, and $\mathcal{V}_{D}=\pi^{D-1} /(D-1)$ ! is the total volume of Hilbert space. The maximum of $g(\phi)$ is located at $\phi=\cos ^{-1}(1 / \sqrt{2(D-1)})$; for large-dimensional Hilbert spaces, $g(\phi)$ is very strongly peaked near the maximum, which is located at $\phi \simeq \pi / 2-$ $1 / \sqrt{2 D}$, very near $\pi / 2$.

As yet a further characterization of the way the vectors are distributed in Hilbert space, we want to define a quantity that indicates how many dimensions of Hilbert space are explored by the vectors in the list $\mathcal{L}$. A possible measure of the number of explored dimensions is the exponential of the entropy, $2^{\Delta H_{\mathcal{S}}}$. This quantity is bounded above by $D$, the dimension of Hilbert space, and gets smaller if the dimensions are occupied with different weights. For example, if two eigenvalues of $\hat{\rho}_{\mathcal{S}}$ are close to $1 / 2$ and all the others are close to zero, then $2^{\Delta H_{\mathcal{S}}} \simeq 2$, indicating that the vectors are essentially confined to a two-dimensional subspace. Unfortunately, if all the vectors in $\mathcal{L}$ are confined to a small sphere with radius $\phi \ll \pi / 2$, $\Delta H_{\mathcal{S}}$ is necessarily small just because all the vectors in the group lie along roughly the same direction in Hilbert space; this is true even if the orthogonal components of the vectors are evenly distributed over all the orthogonal directions in Hilbert space. For example, the density operator describing a uniform distribution of vectors within a sphere of radius $\phi \ll \pi / 2$ has one dominating eigenvalue close to 1 and $D-1$ eigenvalues that are all equal and close to zero [7]. Clearly, in this case $2^{\Delta H_{\mathcal{S}}}$ is not an adequate measure of the number of dimensions explored. On the other hand, if one disregarded the largest eigenvalue in this example, then the exponential of the entropy would become a useful measure of the number of explored dimensions. We therefore introduce the spread $\Delta H_{2}$ as the entropy calculated with the largest eigenvalue of $\hat{\rho}_{\mathcal{S}}$ omitted. The spread is defined as

$$
\Delta H_{2}=-\sum_{k=2}^{D} \frac{\lambda_{k}}{1-\lambda_{1}} \log _{2}\left(\frac{\lambda_{k}}{1-\lambda_{1}}\right),
$$

where $\lambda_{1} \geq \lambda_{2} \geq \ldots \geq \lambda_{D}$ are the eigenvalues of the density operator $\hat{\rho}_{\mathcal{S}}$. By giving different weight to dimensions corresponding to different eigenvalues of $\hat{\rho}_{\mathcal{S}}$, the quantity $\left\lceil 2^{\Delta H_{2}}\right\rceil$ turns 
out to be a good indicator of the number of Hilbert-space dimensions explored by the vectors in the list $\mathcal{L}$, independent of the size of the region occupied by the vectors $(\lceil x\rceil$ denotes the smallest integer greater than or equal to $x$ ). In our analysis of the numerical results, we identify the number of dimensions explored by the entire set of $N$ vectors with the integer $n_{d} \equiv\left\lceil 2^{\Delta H_{2}}\right\rceil$.

To investigate if a quantum map shows hypersensitivity to perturbation, we use the following numerical method. We first compute a list of vectors corresponding to different perturbation histories. Then, for about 50 values of the angle $\phi$ ranging from 0 to $\pi / 2$, we group the vectors in the nearly optimal way described above. Finally, for each grouping and thus for each chosen angle $\phi$, we compute the information $\Delta I(\phi)$ and the entropy $\Delta H(\phi)$. In addition, we compute the angles between all pairs of vectors in the list and plot them as a histogram approximating the distribution function $g(\phi)$.

\section{B. Results for quantum kicked top}

For our numerical study of the kicked top, we use a twist parameter $k=3$ and either spin $J=511.5$ or spin $J=63.5$. The calculations are done in the corresponding 512-dimensional or 64-dimensional even-parity subspace; the even-parity subspace is the eigenspace of the parity (3.4) with eigenvalue +1 . Throughout this section, we consider only two initial states. The first one, the coherent state $|\theta, \varphi\rangle$ with $\theta$ and $\varphi$ given by Eq. (3.6), is centered in a regular region of the classical dynamics; we refer to it as the regular initial state. The second one, referred to as the chaotic initial state, is the coherent state $|\theta, \varphi\rangle$ with $\theta$ and $\varphi$ given by Eq. (3.7); the chaotic initial state is centered in a chaotic region of the classical dynamics.

Figure 11 shows results for spin $J=511.5$ and a total number of $2^{12}=4096$ vectors after $n=12$ perturbed steps. The interaction strength is $g=0.003$, corresponding to perturbing rotations by an angle \pm 0.003 radians about the $z$ axis (the perturbation strength in units of the coherent-state radius $2 J^{-1 / 2}$ is $g J^{1 / 2} / 2=0.034$ ). For Fig. 1(a), the chaotic initial state was used. The distribution of Hilbert-space angles, $g(\phi)$, is concentrated at large angles; i.e., most pairs of vectors are far apart from each other. The information $\Delta I$ needed to track a perturbed vector at resolution level $\phi$ is 12 bits at small angles, where each group contains only one vector. At $\phi \simeq \pi / 16$ the information suddenly drops to 11 bits, which is the information needed to specify one pair of vectors out of $2^{11}$ pairs, the two vectors in each pair being generated by perturbation sequences that differ only at the first step. The sudden drop of the information to 10 bits at $\phi \simeq \pi / 8$ similarly indicates the existence of $2^{10}$ quartets of vectors, generated by perturbation sequences differing only in the first two steps. Figure 1(a) suggests that, apart from the organization into pairs and quartets, there is not much structure in the distribution of vectors for a chaotic initial state. The $2^{10}$ quartets seem to be rather uniformly distributed in a $n_{d}=\left\lceil 2^{\Delta H_{2}}\right\rceil=46$-dimensional Hilbert space, a supposition supported by a comparison with Fig. 2, where results for $2^{13}=8192$ random vectors in 45-dimensional Hilbert space are shown.

The inset in Fig. 11(a) shows the approximate functional dependence of the information needed about the perturbation, $\Delta I_{\min }$, on the tolerable entropy $\Delta H_{\mathrm{tol}}$, based on the data points $\Delta I(\phi)$ and $\Delta H(\phi)$. There is an initial sharp drop of the information, reflecting the grouping of the vectors into pairs and quartets. Then there is a roughly linear decrease of

the information over a wide range of $\Delta H_{\text {tol }}$ values, followed by a final drop with increasing 
slope down to zero at the maximum value of the tolerable entropy, $\Delta H_{\text {tol }}=\Delta H_{\mathcal{S}}$. The large slope of the curve near $\Delta H_{\text {tol }}=\Delta H_{\mathcal{S}}$ can be regarded as a signature of hypersensitivity to perturbation. The linear regime at intermediate values of $\Delta H_{\text {tol }}$ is due to the finite size of the sample of vectors: in this regime the entropy $\Delta H_{r}$ of the $r$ th group is limited by $\log _{2} N_{r}$, the logarithm of the number of vectors in the group. As is discussed in more detail in an upcoming publication [28], finite-sample-size effects dominate the behavior of the curve as long as the number of vectors in a typical group is smaller than the effective dimension of Hilbert space. One therefore expects reduced finite-size effects for larger samples and smaller Hilbert-space dimensions.

To investigate this prediction, we plot in Fig. 3 results for $J=63.5$ and $2^{14}=16384$ vectors after 14 perturbed steps, using an interaction strength $g=0.03\left(\mathrm{gJ}^{1 / 2} / 2=0.12\right)$. As expected, the inset shows that the large-slope behavior of the $\Delta I_{\text {min }}$ vs. $\Delta H_{\text {tol }}$ curve extends to higher values of $\Delta I_{\min }$ than in the previous case. Otherwise, Fig. 3 is very similar to Fig. 四(a). Apart from an obvious organization of the vectors into pairs, there is no apparent structure in the distribution of vectors. The $2^{13}$ pairs of vectors are distributed quasi-randomly in a $n_{d}=45$ dimensional Hilbert space, as is confirmed by comparing Fig. 3 to the case of $2^{13}$ random vectors in 45 dimensions shown in Fig. 2.

We limit the discussion of the regular initial state to spin $J=511.5$ since, as pointed out in Sec. [IIB, only for a large dimension of Hilbert space is the regular initial state well localized inside the regular region around the elliptic fixed point (3.6). Figure 1 1 (b) shows data for $2^{12}$ vectors after 12 perturbed steps in the regular case. The distribution of perturbed vectors starting from the regular initial state is completely different from the chaotic initial condition of Fig. 罒(a). The angle distribution $g(\phi)$ is conspicuously non-random: it is concentrated at angles smaller than roughly $\pi / 4$, and there is a regular structure of peaks and valleys. Accordingly, the information drops rapidly with the angle $\phi$. The number of explored dimensions is $n_{d}=2$, which agrees with results of Peres [8] that show that the quantum evolution in a regular region of the kicked top is essentially confined to a 2-dimensional subspace. The $\Delta I_{\min }$ vs. $\Delta H_{\text {tol }}$ curve in the inset bears little resemblance to the chaotic case. Summarizing, one can say that, in the regular case, the vectors do not get far apart in Hilbert space, explore only few dimensions, and do not explore them randomly.

To obtain better numerical evidence for hypersensitivity in the chaotic case and for the absence of it in the regular case would require much larger samples of vectors, a possibility that is ruled out by restrictions on computer memory and time. The hypothesis most strongly supported by our data is the random character of the distribution of vectors in the chaotic case. To close this section, we give a crude argument to show that randomness in the distribution of perturbed vectors implies hypersensitivity to perturbation. Consider $N$ vectors distributed randomly in a $D$-dimensional Hilbert space, where $N \gg D \gg 1$, so that $\Delta H_{\mathcal{S}} \simeq \log _{2} D$. In this case it was shown in [7] that $\Delta I(\phi) \simeq-D \log _{2}\left(\sin ^{2} \phi\right)$ and $\Delta H(\phi) \simeq \sin ^{2} \phi \log _{2} D \simeq \sin ^{2} \phi \Delta H_{\mathcal{S}}$ if $\Delta I(\phi)<\log _{2} N$. The information is thus proportional to the dimension of Hilbert space, $D$, whereas the tolerable entropy is proportional to $\log _{2} D$. Eliminating $\phi$ gives

$$
\Delta I_{\text {min }} \simeq D \log _{2}\left(\frac{\log _{2} D}{\Delta H_{\mathrm{tol}}}\right) \geq \frac{D}{\ln 2}\left(1-\frac{\Delta H_{\mathrm{tol}}}{\log _{2} D}\right) \simeq \frac{D}{\ln D}\left(\Delta H_{\mathcal{S}}-\Delta H_{\mathrm{tol}}\right) \gg \Delta H_{\mathcal{S}}-\Delta H_{\mathrm{tol}}
$$


i.e., hypersensitivity to perturbation.

\section{QUANTUM MAPS ON THE UNIT SQUARE}

\section{A. Quantized unit square}

In this section, we investigate perturbed quantum maps on the unit square. Our quantization procedure closely follows [29] and [30]. To represent the unit square in $D$-dimensional Hilbert space, we start with unitary "displacement" operators $\hat{U}$ and $\hat{V}$, which produce displacements in the "momentum" and "position" directions, respectively, and which obey the commutation relation [29]

$$
\hat{U} \hat{V}=\hat{V} \hat{U} \epsilon
$$

where $\epsilon^{D}=1$. We choose $\epsilon=e^{2 \pi i / D}$. We further assume that $\hat{V}^{D}=\hat{U}^{D}=\eta= \pm 1$. The case $\eta=1$ corresponds to periodic boundary conditions (used in Sec. VE), whereas the case $\eta=-1$ corresponds to anti-periodic boundary conditions (used in Sections VQ and VD). It follows [29,30] that the operators $\hat{U}$ and $\hat{V}$ can be written as

$$
\hat{U}=e^{2 \pi i \hat{q}} \quad \text { and } \quad \hat{V}=e^{-2 \pi i \hat{p}} .
$$

The "position" and "momentum" operators $\hat{q}$ and $\hat{p}$ both have eigenvalues $(\beta+j) / D$, where the integer $j$ runs over a sequence of $D$ integers and

$$
\beta=\left\{\begin{array}{l}
0, \text { if } \eta=1 \\
\frac{1}{2}, \text { if } \eta=-1
\end{array}\right.
$$

i.e., $\eta=e^{2 \pi i \beta}$.

This quantization procedure fixes the eigenvalues of $\hat{q}$ and $\hat{p}$ to lie within some unit interval, but leaves us free to choose the interval. We use the freedom to write the eigenvalues of $\hat{q}$ and $\hat{p}$ as

$$
q_{j}=\frac{\beta+j}{D}=p_{j}, \quad j=D_{0}, \ldots, D_{0}+D-1,
$$

where the integer $D_{0}$ can be chosen freely. We denote by $\left|q_{j}\right\rangle$ and $\left|p_{j}\right\rangle$ the eigenvectors of $\hat{q}$ and $\hat{p}$ with these eigenvalues. In Sections $\overline{V C}$ and $\overline{V D}$, we choose $D_{0}=0$, corresponding to $q_{j}, p_{j} \in[0,1)$, whereas in Sec. $\nabla \mathrm{E}$, we choose $D_{0}=-D / 2$, corresponding to $q_{j}, p_{j} \in\left[-\frac{1}{2}, \frac{1}{2}\right)$.

In the following, we represent Hilbert-space operators by matrices in the position basis $\left\{\left|q_{j}\right\rangle\right\}$. For consistency of units, let the quantum scale on phase space be $2 \pi \hbar=1 / D$. A transformation between the position basis $\left\{\left|q_{j}\right\rangle\right\}$ and the momentum basis $\left\{\left|p_{j}\right\rangle\right\}$ is effected by the operator $G_{D}$, defined by the matrix elements

$$
\left(G_{D}\right)_{k j}=\left\langle p_{k} \mid q_{j}\right\rangle=\sqrt{2 \pi \hbar} e^{-i p_{k} q_{j} / \hbar}=\frac{1}{\sqrt{D}} e^{-2 \pi i(\beta+k)(\beta+j) / D} .
$$

Following [30], we define a family of $D^{2}$ states $\left|q_{j}, p_{k}\right\rangle\left(j, k=D_{0}, \ldots, D_{0}+D-1\right)$, which we call, in analogy to the continuous case, coherent states. The fiducial coherent state $\left|q_{0}, p_{0}\right\rangle$ 
(assuming $-D<D_{0} \leq 0$ ) is defined as the eigenstate with the smallest eigenvalue of the operator 30

$$
\hat{W}=2-\frac{\hat{U}+\hat{U}^{\dagger}}{2}-\frac{\hat{V}+\hat{V}^{\dagger}}{2}=2-\cos (2 \pi \hat{q})-\cos (2 \pi \hat{p}) .
$$

The other coherent states are defined by applying the displacement operators to the fiducial state, i.e.,

$$
\left|q_{j}, p_{k}\right\rangle=e^{-i \pi j k / D} \hat{U}^{k} \hat{V}^{j}\left|q_{0}, p_{0}\right\rangle=e^{i \pi j k / D} \hat{V}^{j} \hat{U}^{k}\left|q_{0}, p_{0}\right\rangle
$$

The coherent states can also be characterized as minimum-uncertainty states [30].

The coherent states are an overcomplete set, satisfying

$$
\left.\frac{1}{D} \sum_{j, k}\left|q_{j}, p_{k}\right\rangle\left\langle q_{j}, p_{k}\right|=\hat{\mathbb{1}}=\text { (unit operator }\right) .
$$

Therefore they can be used to define a discrete $Q$ function, representing an arbitrary state $|\psi\rangle$ via

$$
Q_{|\psi\rangle}\left(q_{j}, p_{k}\right)=\frac{1}{D}\left|\left\langle q_{j}, p_{k} \mid \psi\right\rangle\right|^{2}
$$

In terms of the $Q$ function, the coherent state $\left|q_{j}, p_{k}\right\rangle$ is represented by roughly circular contours centered at the point $\left(q_{j}, p_{k}\right)$, and it occupies an area of about $1 / D$ [30.

For our discussion of the different quantum maps below, we always assume the initial state $\left|\psi_{0}\right\rangle$ to be a coherent state. The state $\left|\psi_{n}\right\rangle$ after $n$ unperturbed steps is given by

$$
\left|\psi_{n}\right\rangle=\hat{T}^{n}\left|\psi_{0}\right\rangle
$$

where $\hat{T}=\hat{T}_{B}$ for the baker's map (Sec. $\overline{\mathrm{VQ}}$ ), $\hat{T}=\hat{T}_{L}$ for the lazy baker's map (Sec. VD), and $\hat{T}=\hat{T}_{S}$ for the sawtooth and cat maps (Sec. $\left.\nabla \mathrm{E}\right)$.

\section{B. Perturbation operator}

In Sec. IIIC, we presented an example of an interaction with an environment that can be described as a stochastic perturbation of the unitary time evolution. Here we assume from the start that at each step a random perturbation operator is applied following the unperturbed evolution operator $\hat{T}$. The perturbation operator is constructed by analogy to our previous work [4] on the classical baker's map, where the perturbation consisted in shifting the phase-space pattern by a small amount in the $p$ direction. The quantum analogue of this perturbation would be an operator that shifts the $Q$ function of a state by a small amount in the $p$ direction. Due to the discrete nature of our quantum version of the unit square, however, a $Q$ function can be shifted rigidly only if it is shifted by a discrete multiple of $1 / D$.

In order to apply a perturbation that does not shift the momentum by a discrete multiple of $1 / D$, one might consider a perturbation operator $\hat{P}=e^{-2 \pi i \beta \alpha} e^{i \hat{q} \alpha / \hbar}=e^{-2 \pi i \beta \alpha} e^{2 \pi i \hat{q} D \alpha}$ that ramps up the phase uniformly in the position basis, i.e., $P_{k j}=\delta_{k j} e^{-2 \pi i \beta \alpha} e^{2 \pi i q_{j} D \alpha}=\delta_{k j} e^{2 \pi i j \alpha}$. 
When $\alpha$ is not a multiple of $1 / D$, however, this perturbation can couple widely separated momentum eigenstates because of the abrupt phase change between $j=D_{0}+D-1$ and $j=D_{0}$. To avoid this abrupt phase change, we use instead a perturbation operator $\hat{\Pi}$ defined in the position basis by

$$
\hat{\Pi}_{k j}= \begin{cases}\delta_{k j} e^{2 \pi i j \alpha}, & \text { if } D_{0} \leq j<D_{0}+D / 2, \\ \delta_{k j} e^{2 \pi i\left(2 D_{0}+D-j-1\right) \alpha}, & \text { if } D_{0}+D / 2 \leq j<D_{0}+D,\end{cases}
$$

where $\alpha \geq 0$ is the magnitude of the momentum shift ( $D \alpha$ is the magnitude of the momentum shift in units of the separation between momentum eigenstates) and where we restrict attention henceforth to Hilbert spaces with an even dimension $D$. Roughly speaking, the operator $\hat{\Pi}$ shifts the momentum up by $\alpha$ in the left half of the unit square and down by $\alpha$ in the right half of the unit square.

A perturbed time step consists of first applying the unperturbed time-evolution operator $\hat{T}$, followed either by the perturbation operator $\hat{\Pi}$ or by its inverse $\hat{\Pi}^{-1}$, chosen randomly. After $n$ perturbed time steps, the number of different perturbation sequences - or historiesis $2^{n}$, all occurring with equal probability.

\section{Baker's map}

The classical baker's transformation maps the unit square $0 \leq q, p \leq 1$ onto itself according to

$$
(q, p) \longmapsto \begin{cases}\left(2 q, \frac{1}{2} p\right), & \text { if } 0 \leq q \leq \frac{1}{2} \\ \left.2 q-1, \frac{1}{2}(p+1)\right), & \text { if } \frac{1}{2}<q \leq 1\end{cases}
$$

This corresponds to compressing the unit square in the $p$ direction and stretching it in the $q$ direction, while preserving the area, then cutting it vertically, and finally stacking the right part on top of the left part - in analogy to the way a baker kneads dough.

There is no unique way to quantize a classical map. Here we adopt the quantized baker's map introduced by Balazs and Voros [31] and put in more symmetrical form by Saraceno [30]. We assume anti-periodic boundary conditions $\left(\eta=-1, \beta=\frac{1}{2}\right)$ and $D_{0}=0$, which leads, as discussed above, to discrete position and momentum eigenvalues at half-integer values $q_{j}=\left(\frac{1}{2}+j\right) / D=p_{j}, j=0, \ldots, D-1$. The quantum baker's map is now defined by the matrix

$$
T_{B}=G_{D}^{-1}\left(\begin{array}{cc}
G_{D / 2} & 0 \\
0 & G_{D / 2}
\end{array}\right)
$$

where $D$ is even and the matrix elements are to be understood relative to the position basis.

We simulate the perturbed quantum baker's map for two different Hilbert-space dimensions, $D=16$ and $D=256$, but using the same perturbation parameter $\alpha=0.005$ and the same initial coherent state $\left|\psi_{0}\right\rangle=|1 / 2 D, 1 / 2 D\rangle$ (i.e., $j=k=0$ ) in both cases. In units of the separation between $\hat{p}$ eigenstates, this perturbation is 16 times as big for $D=256$ $(D \alpha=1.28)$ as for $D=16(D \alpha=0.080)$.

Figure $1(\mathrm{a})$ shows the results for $D=16$. Because the perturbation is so small, the vectors after 14 perturbed steps are not spread very far apart in Hilbert space, a typical 
angle between vectors being $\phi=\pi / 8$. The information decreases rapidly with resolution angle $\phi$, and the entropy $\Delta H_{\mathcal{S}}$ is less than 1 bit. Nevertheless, the data have several features characteristic of chaos: (i) no structure is apparent in the distribution function $g(\phi)$, indicating a random distribution of vectors within the small region explored by the vectors; (ii) even though the entropy $\Delta H_{\mathcal{S}}$ is very small, the number of explored dimensions is $n_{d}=6$; (iii) although the $\Delta I_{\min }$ vs. $\Delta H_{\text {tol }}$ curve in the inset is qualitatively different from the chaotic cases in Figs. 11 and 3, it shows nonetheless that in order to keep the entropy increase $\Delta H_{\text {tol }}$ below $10 \%$ of its maximum value $\Delta H_{\mathcal{S}}$ requires at least 12 bits of information about the perturbation. These three features should be contrasted with the corresponding behavior for the regular initial state of the quantum kicked top [Fig. 1(b)].

The effects of the perturbation become more pronounced if the Hilbert-space dimension is increased to $D=256$, as can be seen in Fig. $4(\mathrm{~b})$, where results for $2^{13}$ vectors after 13 perturbed steps are plotted. Here, besides an organization into pairs of vectors a distance of about $3 \pi / 16$ apart, the distribution of the vectors is similar to the distribution of random vectors in Fig. 2. The vectors explore $n_{d}=111$ Hilbert-space dimensions, and the $\Delta I_{\text {min }}$ vs. $\Delta H_{\text {tol }}$ curve in the inset has a steep slope near $\Delta H_{\text {tol }}=\Delta H_{\mathcal{S}}$, a signature of hypersensitivity to perturbation.

\section{Lazy baker's map}

The lazy baker's transformation [32] maps the unit square $0 \leq q, p \leq 1$ onto itself according to

$$
(q, p) \longmapsto \begin{cases}\left(3 q, \frac{1}{3} p\right), & \text { if } 0 \leq q \leq \frac{1}{3} \\ (1-p, q), & \text { if } \frac{1}{3}<q \leq \frac{2}{3} \\ \left(3 q-2, \frac{1}{3}(p+2)\right), & \text { if } \frac{2}{3}<q \leq 1\end{cases}
$$

Here the unit square is cut into three vertical stripes, the left and right stripes are compressed in the $p$ direction and stretched in the $q$ direction by a factor of 3 , the middle stripe is rotated by $90^{\circ}$, and finally the three parts are stacked on top of each other - somehow imitating the action of a lazy baker who neglects to knead the middle portion of the dough.

To define a quantized lazy baker's map, the unit square is quantized in the same way as for the ordinary baker's map $\left(\eta=-1\right.$ and $\left.D_{0}=0\right)$. The dimension $D$ of Hilbert space is chosen to be a multiple of 6 . The quantum-mechanical time-evolution operator is [32]

$$
T_{L}=G_{D}^{-1}\left(\begin{array}{ccc}
G_{D / 3} & 0 & 0 \\
0 & I_{D / 3} & 0 \\
0 & 0 & G_{D / 3}
\end{array}\right)
$$

where $I_{D / 3}$ is the identity matrix in $D / 3$ dimensions.

The quantum lazy baker's map was introduced [32] because it allows investigation of regular as well as chaotic evolution, while being almost as simple as the ordinary baker's map. As we did for the kicked top, we compare regular and chaotic evolution by choosing different initial vectors. We use a Hilbert-space dimension $D=216$ - even multiples of powers of 3 reflect best the symmetries of the map [32 - and a perturbation parameter 
$\alpha=0.005(D \alpha=1.08)$ to facilitate comparison with the results of the last section for the ordinary baker's map.

For the chaotic case shown in Fig. 5(a), we choose the initial state to be the coherent state $\left|\psi_{0}\right\rangle=|52.5 / 216,52.5 / 216\rangle$ (i.e., $j=k=52$ ). The $Q$ function of this state, of diameter $\simeq 1 / \sqrt{216} \simeq 1 / 15$, covers a region containing a large number of hyperbolic points separating regular regions in a fractal hierarchy [32]; thus one expects chaotic behavior for this initial condition 32. Indeed, Fig. 5(a), similarly to Fig. W(a), shows that after 13 steps the $2^{13}$ vectors are distributed quasi-randomly, aside from an organization into pairs of vectors. The vectors explore $n_{d}=136$ dimensions, and the $\Delta I_{\min }$ vs. $\Delta H_{\text {tol }}$ curve has the shape that seems to be characteristic for chaos.

The results for the regular case, shown in Fig. 5(b), are strikingly different. For this simulation, we choose the initial state to be the coherent state that is specified by $j=k=108$ (i.e., $\left|\psi_{0}\right\rangle=|108.5 / 216,108.5 / 216\rangle$ ). This state, also of diameter $1 / \sqrt{216} \simeq 1 / 15$, is almost entirely confined to the central square of side length $1 / 3$, which is simply rotated by $90^{\circ}$ at each step. One expects regular behavior for this trivial time evolution, and Fig. 5(b) confirms this expectation. The peaks and valleys of the distribution function $g(\phi)$ indicate a regular spacing between the vectors, the vectors explore only $n_{d}=2$ dimensions, and the $\Delta I_{\min }$ vs. $\Delta H_{\text {tol }}$ curve has the shape that we encountered in Fig. 1 (b), which seems to be characteristic for regular dynamics.

\section{E. Sawtooth and cat maps}

The classical sawtooth map 33 on the torus $q, p \in\left[-\frac{1}{2}, \frac{1}{2}\right)$ is given by

$$
(q, p) \longmapsto((q+p+K q) \bmod 1,(p+K q) \bmod 1)
$$

where the sawtooth parameter $K$ is an arbitrary real number and mod 1 is taken relative to the relevant interval $\left[-\frac{1}{2}, \frac{1}{2}\right)$. For $K=0$, the map is trivial and completely regular, describing a free rotor. For $K>0$, the map is completely hyperbolic (chaotic). For $K=1$, it becomes the Arnol'd cat map [34].

For the quantized version of the sawtooth maps [35, 36], we assume periodic boundary conditions $(\eta=1, \beta=0)$ and $D_{0}=-D / 2$, which leads to discrete position and momentum eigenvalues at $q_{j}=p_{j}=j / D, j=-D / 2, \ldots, D / 2-1$, where $D$ is even. The quantum evolution operator is given by the matrix elements [35, 36.

$$
\left(T_{S}\right)_{j k}=\left\langle q_{j}\left|\hat{T}_{S}\right| q_{k}\right\rangle=\frac{e^{-i \pi / 4}}{\sqrt{D}} e^{i \pi K k^{2} / D} e^{i \pi(j-k)^{2} / D} .
$$

For the simulation of the perturbed sawtooth map, we use the Hilbert-space dimension $D=256$, a very small perturbation parameter $\alpha=0.001(D \alpha=0.256)$, and the initial coherent state $\left|\psi_{0}\right\rangle=|0,0\rangle$. We compare the distribution of $2^{13}$ vectors obtained after 13 perturbed steps for three different values of the sawtooth parameter.

For Fig. 6(a) we make a generic choice of the sawtooth parameter, $K=0.5$, corresponding to chaos in the classical map. As in the behavior found for the 16-dimensional baker's map in Fig. Aa, the very small perturbation strength keeps the vectors from moving very far apart. Nevertheless, the vectors explore $n_{d}=38$ dimensions, and to keep the entropy increase 
to $10 \%$ of its maximum value requires nearly 12 bits. Altogether, as for the baker's map in 16 dimensions, we come to the conclusion that the sawtooth map with $K=0.5$ shows characteristics of hypersensitivity to perturbation.

In contrast to the generic sawtooth map, consider the free rotor resulting from a sawtooth parameter $K=0$, a case illustrated in Fig. 6(b). The free rotor shows entirely regular behavior. Although the perturbation manages to move some vectors far apart from each other, as is evident from the shape of the distribution function $g(\phi)$, the vectors explore only $n_{d}=2$ dimensions of Hilbert space. The $\Delta I_{\min }$ vs. $\Delta H_{\text {tol }}$ curve has the shape already found in the regular cases of the kicked top and the lazy baker's map.

Finally, Fig. 7 shows results for the Arnol'd cat map, generated by a sawtooth parameter $K=1$. Integer values of the sawtooth parameter $K$ are non-generic in the sense that they give the map additional symmetries [33,35,36]. These additional symmetries might be responsible for the structure in the angle distribution $g(\phi)$, apparent in Fig. 7, which is absent in the generic case shown in Fig. 6(a). Another difference between the cat map and the generic sawtooth map, which might also be due to the additional symmetries, is the grouping into pairs, quartets, and possibly octets, revealed by the sudden drops in the $\Delta I$ vs. $\phi$ curve in Fig. 7, but which is absent in Fig. 6(a). As already discussed for the kicked top in Sec. IVB, this grouping shows up in the $\Delta I_{\text {min }}$ vs. $\Delta H_{\text {tol }}$ plot as an an initial drop of the information. Apart from this initial drop, however, the $\Delta I_{\min }$ vs. $\Delta H_{\text {tol }}$ curve is similar to the generic case, indicating that the quantum cat map, like the generic sawtooth map, is hypersensitive to perturbation.

\section{CONCLUSION}

Our aim in this paper is to explore, via numerical simulations, the notion of hypersensitivity to perturbation in quantum systems, trying to determine whether hypersensitivity might provide a criterion, as it does for classical systems, for distinguishing regular from chaotic quantum dynamics. What has been learned? Our simulations indicate that hypersensitivity is a valuable tool for investigating chaotic quantum dynamics. Our key finding is that if a chaotic quantum system is perturbed stochastically, the different perturbation histories tend to produce state vectors that are distributed randomly over a large number of Hilbert-space dimensions, whereas if a regular quantum system is subjected to a similar perturbation, the resulting state vectors explore only a few Hilbert-space dimensions and do not explore even those dimensions randomly.

The nearly random distribution of vectors translates into a characteristic $\Delta I_{\text {min }}$ vs. $\Delta H_{\text {tol }}$ curve, evident in the insets of Figs. 11(a), 2, 3, 田(b), and 5(a), which seems to be a property of quantum chaos. This characteristic curve is distinctly different from the $\Delta I_{\text {min }}$ vs. $\Delta H_{\text {tol }}$ curve produced by regular quantum dynamics [Figs. 11(b), 5(b), and 6(b)]. After an initial sharp drop due to perturbation histories that differ only at the first few steps, the $\Delta I_{\min }$ vs. $\Delta H_{\text {tol }}$ curve for chaotic dynamics has a region of roughly linear decrease that extends over a broad range of values of $\Delta H_{\text {tol }}$; this linear decrease is a consequence of the finite sample of vectors produced by the perturbation (always a binary perturbation in our simulations) and thus cannot be said to be a property of the chaotic dynamics. The most important part of the $\Delta I_{\text {min }}$ vs. $\Delta H_{\text {tol }}$ curve is the steep increase in $\Delta I_{\min }$ as $\Delta H_{\text {tol }}$ is decreased just below its maximum value of $\Delta H_{\mathcal{S}}$. This steep increase means the following: to decrease the entropy a 
small amount below the entropy that comes from averaging over the stochastic perturbation requires a large amount of information about the perturbing environment, much larger than the entropy decrease. This rapid increase in information is what we call hypersensitivity to perturbation. It seems to be a feature of chaotic quantum dynamics.

These considerations set the stage for further work: to understand in detail the steep rise in the $\Delta I_{\min }$ vs. $\Delta H_{\text {tol }}$ curve and how it is cut off by a finite sample of vectors; to show that quantum hypersensitivity to perturbation is indeed a property of chaotic quantum dynamics and not an artifact of particular perturbations; to study the time dependence of quantum hypersensitivity and perhaps to extract from the time dependence a quantity like the Kolmogorov-Sinai entropy of classical chaotic dynamics; and, finally, to develop a general theory of quantum hypersensitivity to perturbation, which would allow one to relate it to other properties of chaotic quantum dynamics.

\section{ACKNOWLEDGMENTS}

The authors profited from discussions with H. Barnum, D. Steinbach, and R. Menegus. The authors thank the Aspen Center for Physics for making available its uniquely productive research environment during the period when this work was first conceived and again when it was being completed.

\section{APPENDIX A:}

Equation (2.5) is true for any measurement on the environment that is described by a set of completely positive operations $\mathcal{F}_{r}$; an operation is a linear, trace-decreasing mapping that takes positive operators to positive operators and, hence, takes a density operator to an (unnormalized) post-measurement density operator [17]. The joint state of the system and the environment after a measurement that yields results $r$ is

$$
\frac{\mathcal{F}_{r}\left(\hat{\rho}_{\text {total }}\right)}{p_{r}}=\frac{\sum_{i} \hat{A}_{r i} \hat{\rho}_{\text {total }} \hat{A}_{r i}^{\dagger}}{p_{r}} .
$$

The complete positivity of $\mathcal{F}_{r}$ means that it can be written as the sum involving the operators $\hat{A}_{r i}$ [17]; since we are considering a measurement on the environment, these operators are environment operators. The probability to obtain measurement result $r$ becomes

$$
p_{r}=\operatorname{tr}\left(\mathcal{F}_{r}\left(\hat{\rho}_{\text {total }}\right)\right)=\operatorname{tr}\left(\hat{\rho}_{\text {total }} \hat{E}_{r}\right)
$$

where

$$
\hat{E}_{r}=\sum_{i} \hat{A}_{r i}^{\dagger} \hat{A}_{r i}
$$

is an element of a POVM. Completeness of the POVM guarantees that

$$
\left.\sum_{r} \hat{E}_{r}=\hat{\mathbb{1}}_{\mathcal{E}}=\text { (environment unit operator }\right) \text {. }
$$

The system state after a meaurement that yields result $r$ is obtained by tracing out the environment, 


$$
\hat{\rho}_{r}=\frac{\operatorname{tr}_{\mathcal{E}}\left(\mathcal{F}_{r}\left(\hat{\rho}_{\text {total }}\right)\right)}{p_{r}}=\frac{\operatorname{tr}_{\mathcal{E}}\left(\hat{\rho}_{\text {total }} \hat{E}_{r}\right)}{p_{r}}
$$

The crucial step here uses the cyclic property of the trace to move the operators $\hat{A}_{r i}$ to the right side of the trace; this step is allowed within an environment trace because these operators are, by assumption, environment operators. The important content of Eq. (A1) is that even though there are many operations $\mathcal{F}_{r}$ that are consistent with the POVM $\hat{E}_{r}$, the system state after a measurement on the environment depends only on the POVM. Equation (2.5) follows from summing over $r$ and using the completeness of the POVM:

$$
\sum_{r} p_{r} \hat{\rho}_{r}=\operatorname{tr}_{\mathcal{E}}\left(\hat{\rho}_{\text {total }}\right)=\hat{\rho}_{\mathcal{S}}
$$




\section{REFERENCES}

* Present address: Department of Physics, Queen Mary and Westfield College, University of London, Mile End Road, London E1 4NS, UK (e-mail: r.schack@qmw.ac.uk)

[1] M. V. Berry, Proc. Roy. Soc. Lond. A 413, 183 (1987).

[2] M. V. Berry, in New Trends in Nuclear Collective Dynamics, edited by Y. Abe, H. Horiuchi, and K. Matsuyanagi (Springer, Berlin, 1992), p. 183.

[3] C. M. Caves, in Physical Origins of Time Asymmetry, edited by J. J. Halliwell, J. PérezMercader, and W. H. Zurek (Cambridge University Press, Cambridge, England, 1993), p. 47.

[4] R. Schack and C. M. Caves, Phys. Rev. Lett. 69, 3413 (1992).

[5] R. Schack and C. M. Caves, submitted to Phys. Rev. E [e-print chao-dyn/9506002].

[6] R. Schack and C. M. Caves, Phys. Rev. Lett. 71, 525 (1993).

[7] R. Schack, G. M. D’Ariano, and C. M. Caves, Phys. Rev. E 50, 972 (1994).

[8] A. Peres, in Quantum Chaos, edited by H. A. Cerdeira, R. Ramaswamy, M. C. Gutzwiller, and G. Casati (World Scientific, Singapore, 1991), p. 73.

[9] A. Peres, Quantum Theory: Concepts and Methods (Kluwer Academic Publishers, Dordrecht, The Netherlands, 1993).

[10] R. Landauer, IBM J. Res. Develop. 5, 183 (1961).

[11] R. Landauer, Nature 355, 779 (1988).

[12] G. J. Chaitin, Algorithmic Information Theory (Cambridge University Press, Cambridge, England, 1987).

[13] W. H. Zurek, Nature 341, 119 (1989).

[14] W. H. Zurek, Phys. Rev. A 40, 4731 (1989).

[15] C. M. Caves, in Complexity, Entropy, and the Physics of Information, edited by W. H. Zurek (Addison Wesley, Redwood City, California, 1990), p. 91.

[16] R. Schack, submitted to Phys. Rev. E [e-print hep-th/9409022].

[17] K. Kraus, States, Effects, and Operations: Fundamental Notions of Quantum Theory (Springer, Berlin, 1983).

[18] H. Frahm and H. J. Mikeska, Z. Phys. B 60, 117 (1985).

[19] F. Haake, M. Kuś, and R. Scharf, Z. Phys. B 65, 381 (1987).

[20] G. M. D’Ariano, L. R. Evangelista, and M. Saraceno, Phys. Rev. A 45, 3646 (1992).

[21] J. M. Radcliffe, J. Phys. A 4, 313 (1971).

[22] P. W. Atkins and J. C. Dobson, Proc. Roy. Soc. Lond. A 321, 321 (1971).

[23] A. M. Perelomov, Generalized Coherent States (Springer, Berlin, 1986).

[24] W. K. Wootters, Phys. Rev. D 23, 357 (1981).

[25] A. M. Perelomov, Commun. Math. Phys. 26, 222 (1972).

[26] R. Balian, From Microphysics to Macrophysics, Vol. 1 (Springer, Berlin, 1991).

[27] C. M. Caves and P. D. Drummond, Rev. Mod. Phys. 66, 481 (1994).

[28] H. Barnum, R. Schack, and C. M. Caves, in preparation.

[29] H. Weyl, The Theory of Groups and Quantum Mechanics (Dover, New York, 1950).

[30] M. Saraceno, Ann. Phys. 199, 37 (1990).

[31] N. L. Balazs and A. Voros, Ann. Phys. 190, 1 (1989).

[32] A. Lakshminarayan and N. L. Balazs, Ann. Phys. 226, 350 (1993).

[33] I. C. Percival and F. Vivaldi, Physica D 27D, 373 (1987). 
[34] V. I. Arnold and A. Avez, Ergodic Problems of Classical Mechanics (Benjamin, New York, 1968).

[35] A. Lakshminarayan and N. L. Balazs, to be published [e-print chao-dyn/9307005].

[36] A. Lakshminarayan, Phys. Lett. A 192, 345 (1994). 


\section{FIGURES}

FIG. 1. Results characterizing the distribution of Hilbert-space vectors for the perturbed kicked top with $J=511.5, k=3$, and $g=0.003$ after $n=12$ time steps. The two main diagrams show, as a function of the angle $\phi$, the distribution $g(\phi)$ of Hilbert-space angles (unnormalized, in arbitrary units), the average information $\Delta I(\phi)$ to specify a vector at the resolution given by $\phi$ (in bits), and the average conditional entropy $\Delta H(\phi)$ (in bits). See the text for a precise definition of these quantities. In the insets, $\Delta I(\phi)$ is plotted versus $\Delta H(\phi)$ using the same data points as in the main diagrams. The labeling of the inset axes is motivated by the fact that $\Delta I(\phi)$ is a good approximation to $\Delta I_{\min }$ for $\Delta H_{\text {tol }}=\Delta H(\phi)$. (a) Chaotic case, i.e., initial coherent state $\left|\psi_{0}\right\rangle=|\theta, \varphi\rangle$ centered in the chaotic region with $\theta$ and $\varphi$ given by Eq. (3.7). Distribution of all $2^{12}$ vectors generated after 12 perturbed steps. (b) Regular case, i.e., initial coherent state centered at the elliptic fixed point given by Eq. (3.6). All $2^{12}$ vectors generated after 12 perturbed steps.

FIG. 2. The same quantities as in Fig. 1 plotted using a list of $2^{13}$ random vectors in 45-dimensional Hilbert space.

FIG. 3. As Fig. 11(a) (chaotic case), but using $J=63.5, g=0.03$, and all $2^{14}$ vectors generated after 14 perturbed steps.

FIG. 4. As Fig. 1, but for the quantum baker's map with initial coherent state $\left|\psi_{0}\right\rangle=|1 / 2 D, 1 / 2 D\rangle$. (a) $D=16, \alpha=0.005$, all $2^{14}$ vectors after 14 steps. (b) $D=256$, $\alpha=0.005$, all $2^{13}$ vectors after 13 steps.

FIG. 5. As Fig. 1, but for the quantum lazy baker's map. $D=216, \alpha=0.005$, all $2^{13}$ vectors after 13 steps. (a) Chaotic case, initial coherent state $\left|\psi_{0}\right\rangle=|52.5 / 216,52.5 / 216\rangle$. (b) Regular case, initial coherent state $\left|\psi_{0}\right\rangle=|108.5 / 216,108.5 / 216\rangle$.

FIG. 6. As Fig. 1, but for the quantum sawtooth maps. Initial coherent state $\left|\psi_{0}\right\rangle=|0,0\rangle$, $D=256, \alpha=0.001$, all $2^{13}$ vectors after 13 steps. (a) Chaotic sawtooth map, $K=0.5$. (b) Regular free rotor, $K=0$.

FIG. 7. As Fig. 1, but for the quantum cat map, i.e., the quantum sawtooth map with $K=1$. Initial coherent state $\left|\psi_{0}\right\rangle=|0,0\rangle, D=256, \alpha=0.001$, all $2^{13}$ vectors after 13 steps. 

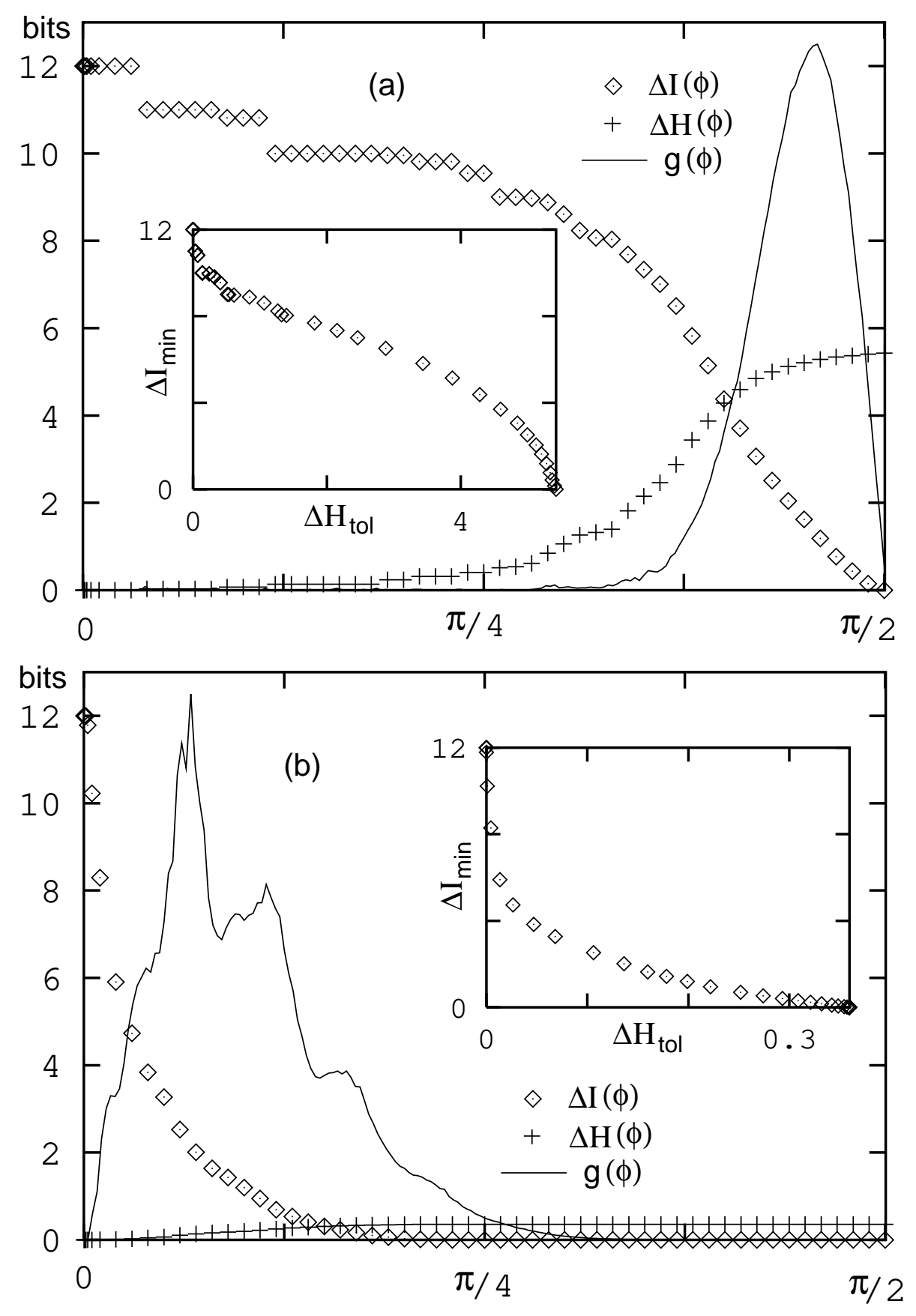

Hilbert-space angle $\phi$

Figure 1 


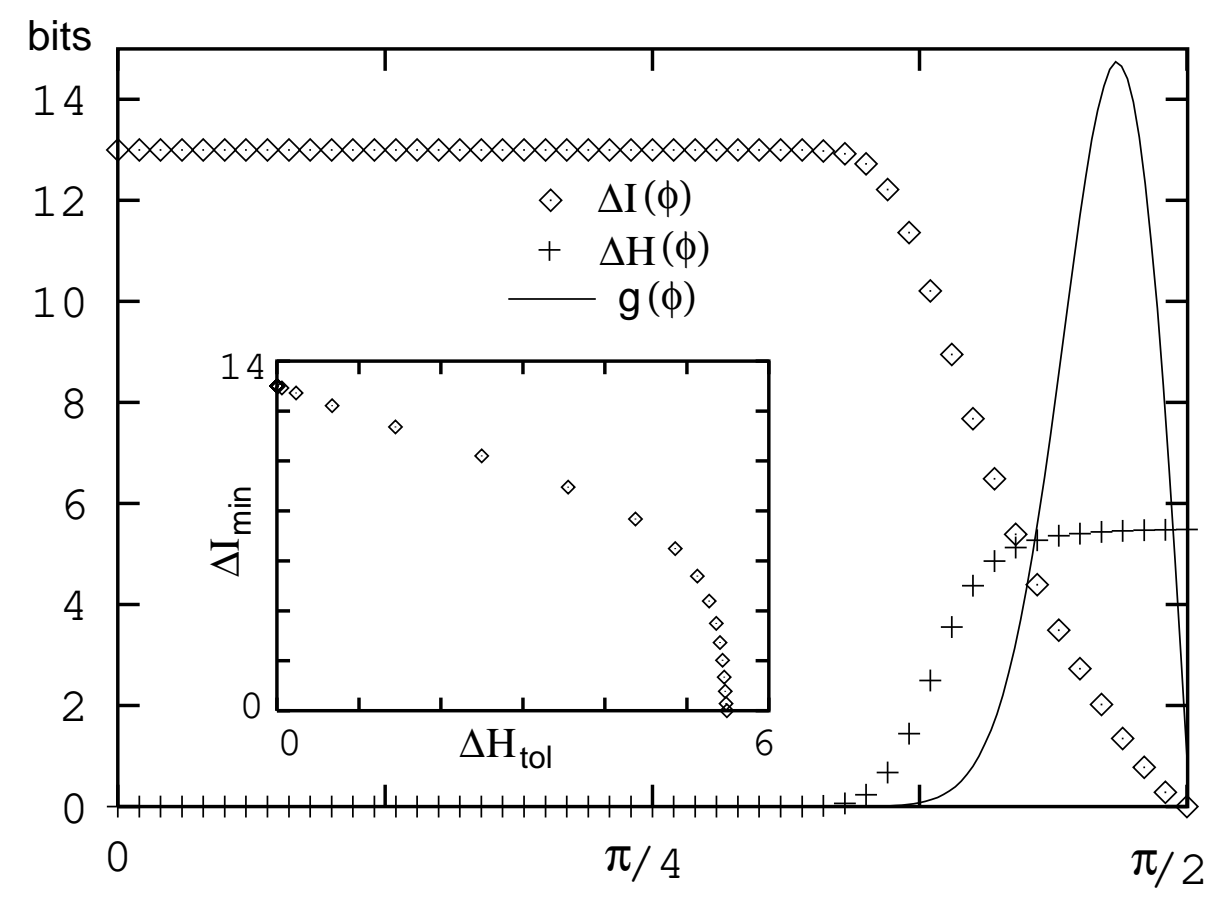

Hilbert-space angle $\phi$

Figure 2 


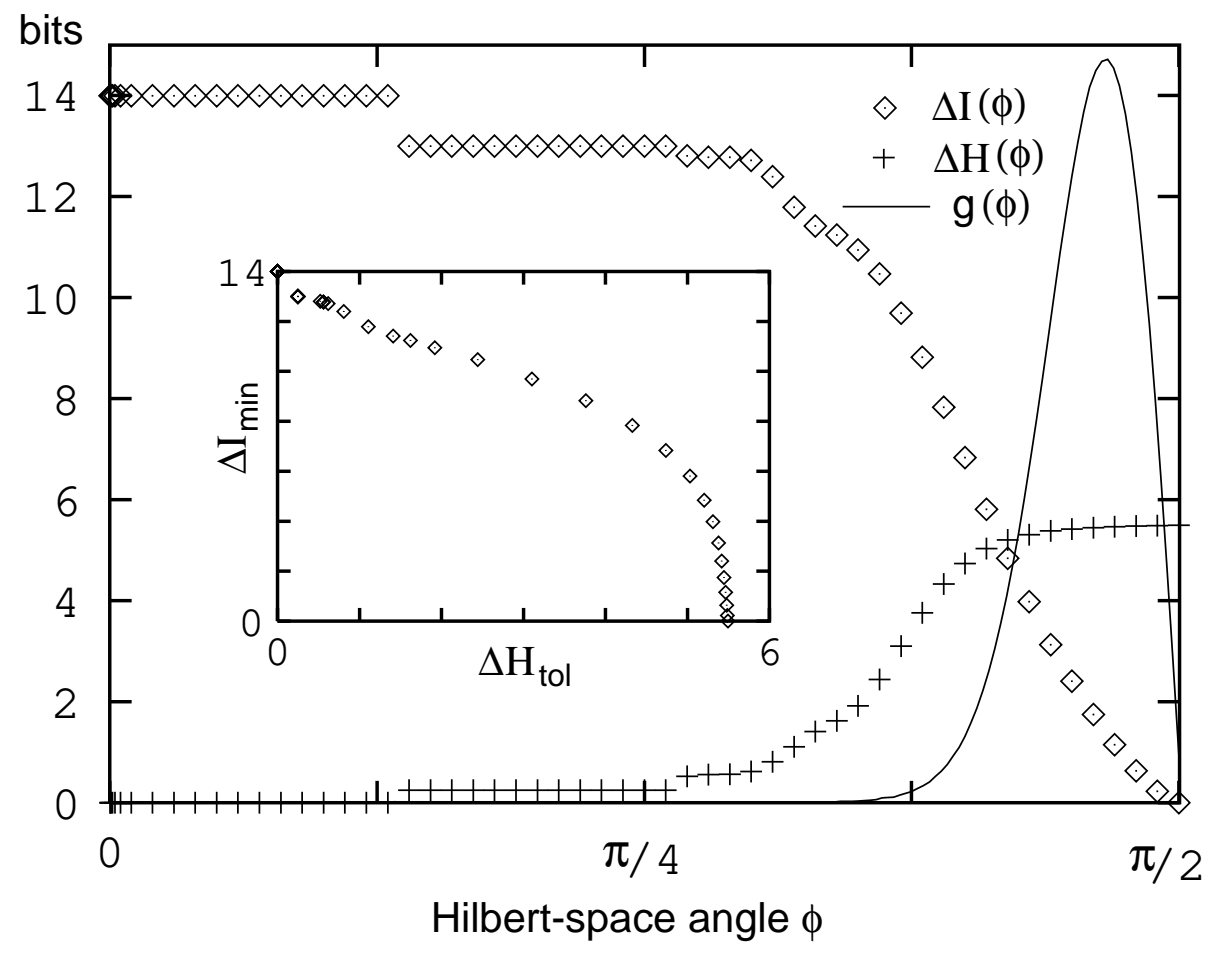

Figure 3 

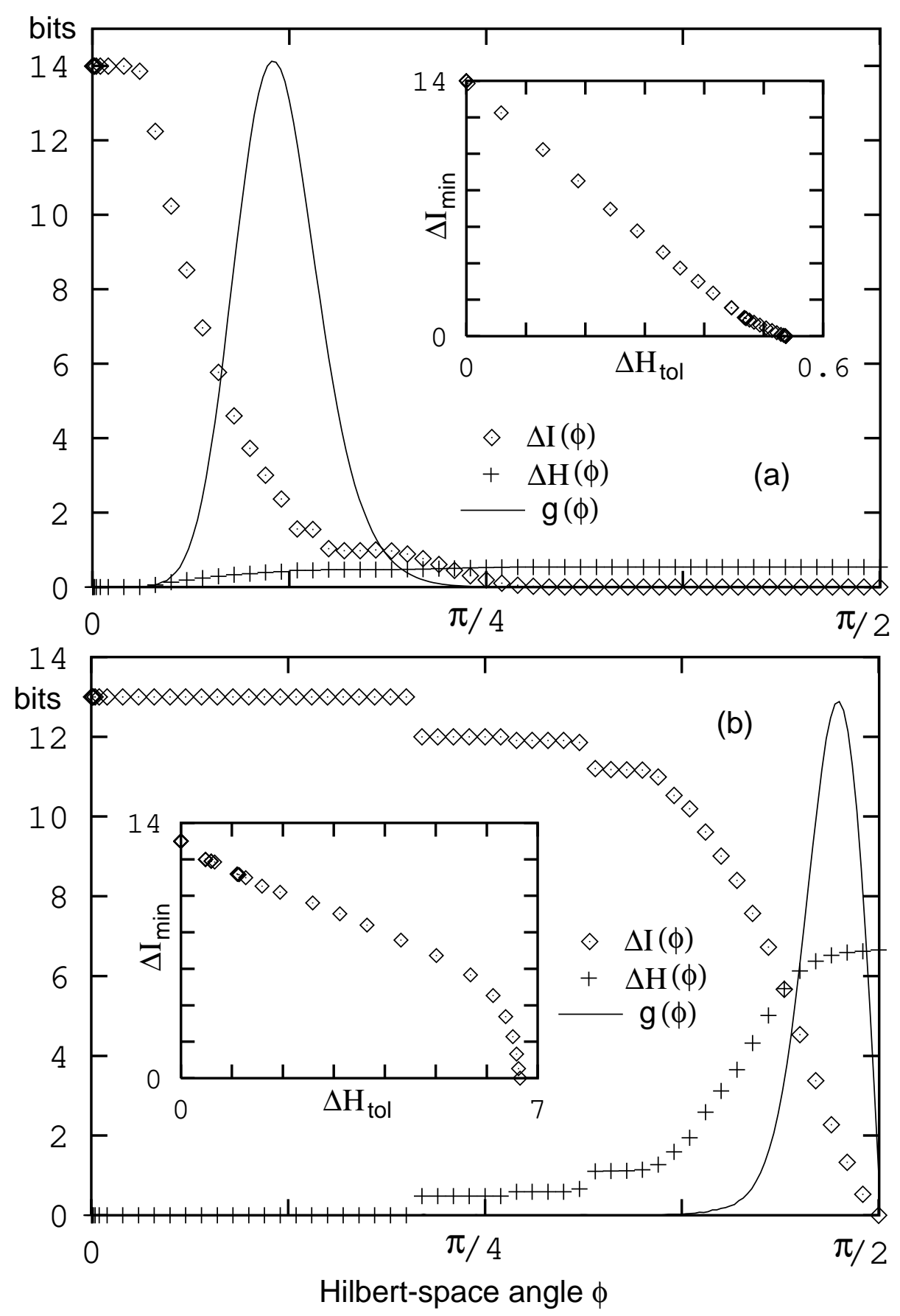

Figure 4 

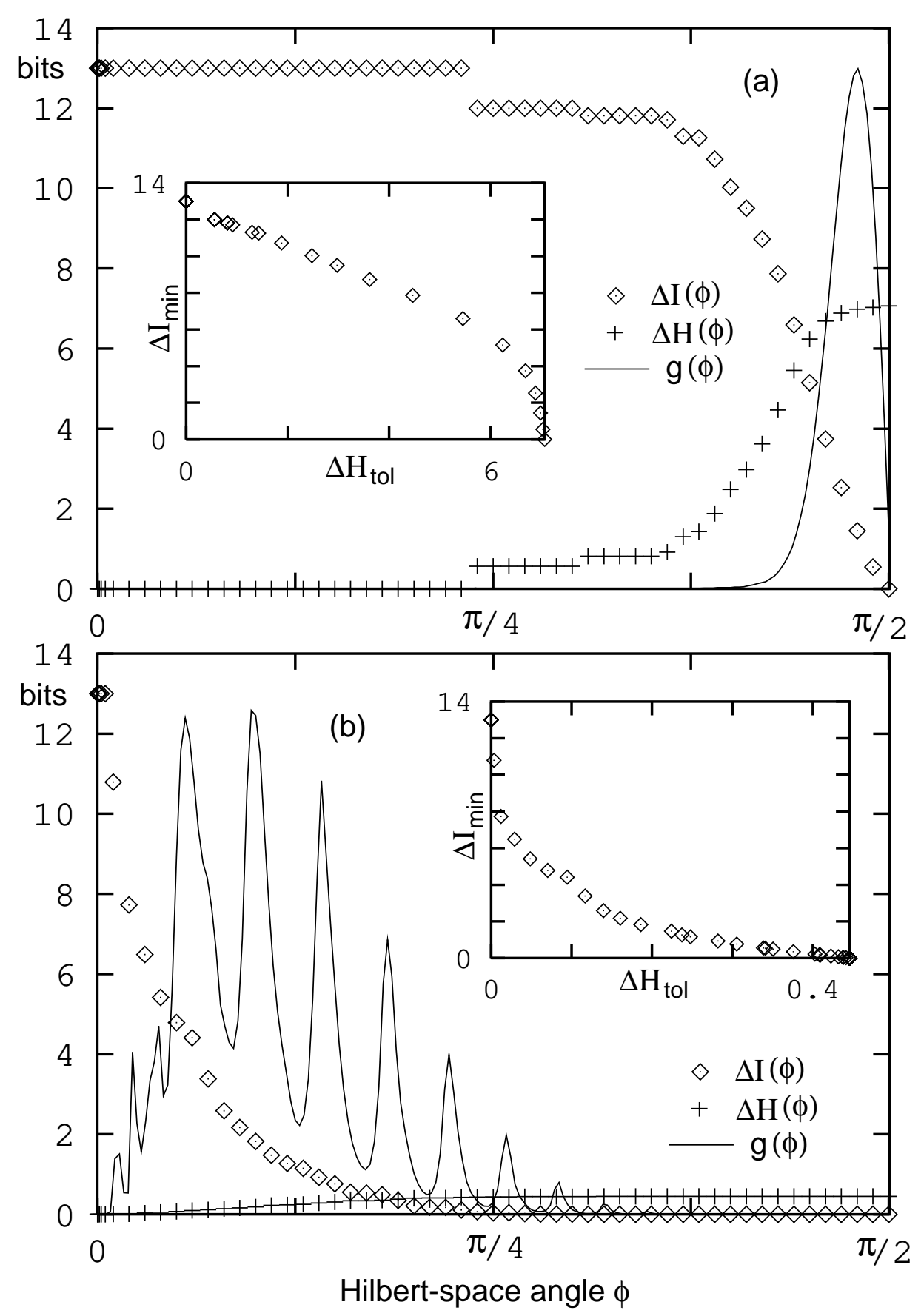

Figure 5 

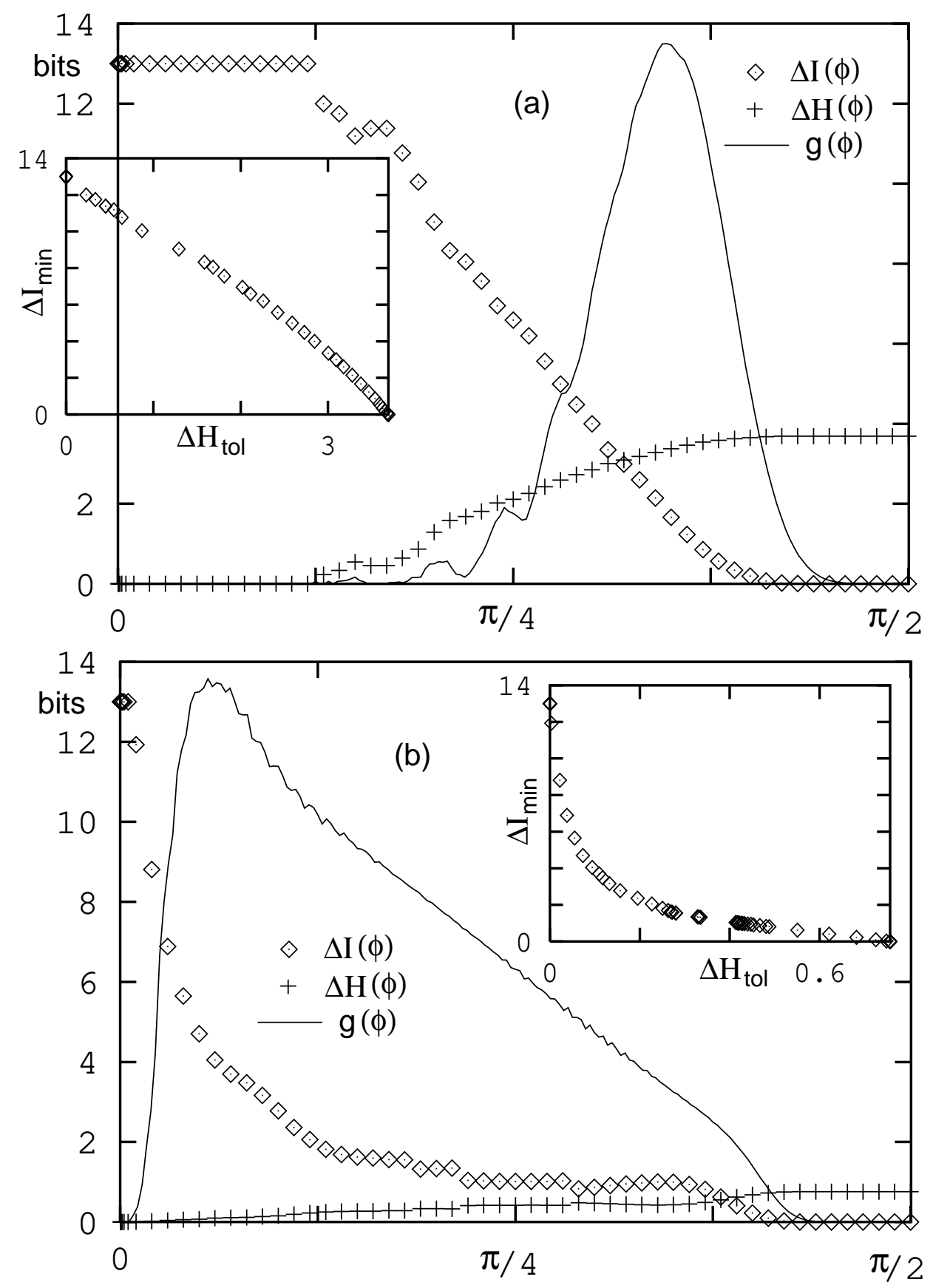

Hilbert-space angle $\phi$

Figure 6 


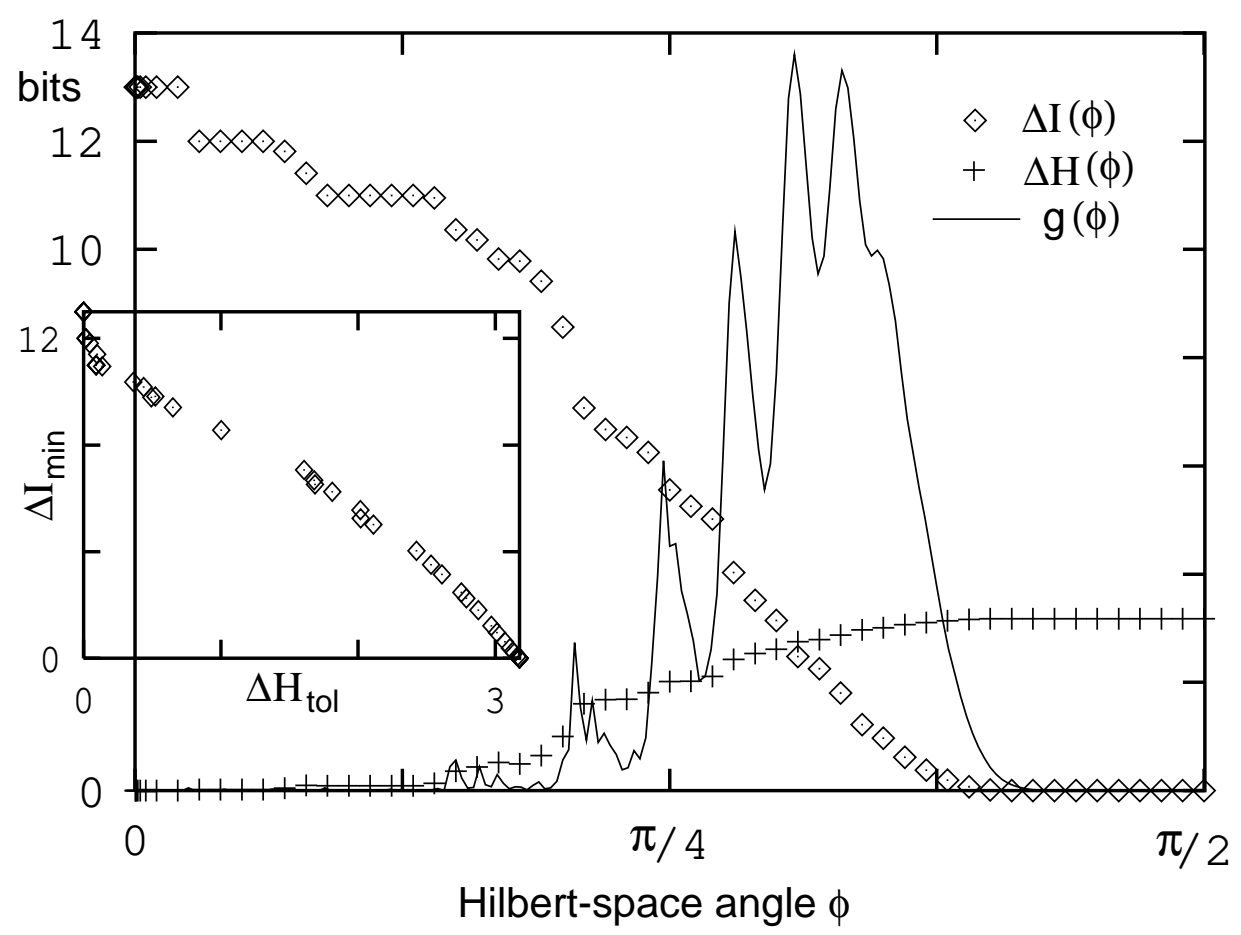

Figure 7 